\title{
Research Paper \\ The Effect of one Year of Inactivity Caused by Covid-19 on the Motor Func- tion of the Elderly Living in Care Centers
}

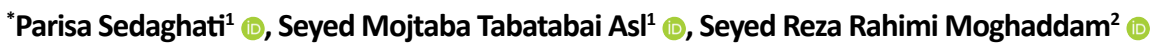

1. Department of Sport Injuries and Corrective Exercise, Faculty of Physical Education and Sport Sciences, University of Guilan, Rasht, Iran.

2. Research and Technology Council of the General Administration of Sport and Youth of Guilan Province, Rasht, Iran.

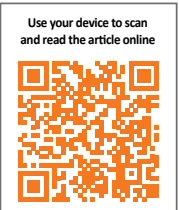

Citation Sedaghati P, Tabatabai AsI SM, Rahimi Moghaddam SR. The Effect of one Year of Inactivity Caused by Covid-19 on the Motor Function of the Elderly Living in Care Centers. Scientific Journal of Rehabilitation Medicine. 2021; 10(3):436-445. https://doi. org/10.32598/sjrm.10.3.5

doi ${ }^{\circ} h$ ttps://doi.org/10.32598/sjrm.10.3.5

Received: 10 May 2021 Accepted: 14 Jun 2021 Available Online: 23 Jul 2021

Keywords:

Coronavirus, Balance posture, Motor function, Falling probability

\begin{abstract}
Background and Aims Although the outbreak of coronavirus has caused problems in all aspects of human life. Still, this disease has caused severe damage to the health of the elderly community and the level of physical activity and free movement of the elderly. This study aimed to investigate the effect of one year of coronavirus limitation on the motor function of the elderly living in care centers.

Methods The present study was a cohort study that was conducted in the field. The statistical population of this study was male and female elderly over 60 years of age from two nursing homes in Rash City. Berg functional balance tests evaluated the motor function of the elderly, Timed Up and Go (TUG) Test or fall probability test and walking speed test or 10-meter walk test. Shapiro-Wilk test was used to check the normality of the data, descriptive statistics were used to determine the mean and standard deviation, and paired t-test and McNemar was used to analyze the data and examine the intra-group differences between the data in SPSS software version 26.

Results The results of the correlated t-test showed that the time up and go test in the elderly under the influence of one year of movement limitation in the post-coronary period had a significant increase $(P=0.001)$, also in functional balance index $(P=0.03)$ significant changes according to For a year, the elderly had limited mobility. There was a significant decrease in the walking variable $(P=0.001)$ following this period. It also showed a significant increase in the probability of falling index $(P=0.021)$.

Conclusion According to the findings of the present study, elderly cares centers and families with the elderly should pay close attention to the need for a level of physical activity and exercise in this critical period of coronation for these people and in addition to planning to increase the level of physical activity These people, they are encouraged to participate in physical activity.
\end{abstract}

\section{Extended Abstract}

\section{Introduction}

ld age is a critical period of life. Considering the special needs of this period, paying attention to the quality of life and physical activity in the elderly is considered very important and should not be ignored. With age, physiological dysfunctions develop that become more prominent in the sixth decade of life. Various studies show that with age, due to disorders in multiple systems of the body, primarily due to reduced physical ac-

\section{*Corresponding Author:}

Parisa Sedaghati, PhD.

Address: Department of Sport Injuries and Corrective Exercise, Faculty of Physical Education and Sport Sciences, University of Guilan, Rasht, Iran. Tel: +98 (912) 2768326

E-Mail: sedaghati@guilan.ac.ir 
tivity, the ability of older people to maintain balance and physical fitness decreases and leads to an increased risk of falling. Several decades of living, physiological and functional abilities such as muscle strength, nerve function, lung function, cardiovascular function, body composition, and Bone Mass Index (BMI) decline. Imbalance is one of the main problems in the elderly. Changes in the body's physical condition and biological processes in old age are associated with decreased performance in flexibility, agility, speed, and balance, so older people are more at risk for falls than those in other age groups.

The elderly are at an age when they are always in need of social activities, and sports activities can meet their social, physical, and psychological needs to a great extent. Progressive changes at the community level have led to the inactive form of various forms of physical activity and leisure, which has led to the formation of an inactive lifestyle.

Meanwhile, some vulnerable groups, including the elderly, have suffered the most from lifestyle changes. The occurrence of some international crises has always affected the level of physical activity of the elderly. Meanwhile, the outbreak of Covid-19 as an international problem has caused severe damage to the physical fitness and exercise of the elderly worldwide.

\section{Methods}

This study was carried out as a cohort study. The statistical population of this study was $<60$ years elderly males and females from two care centers in Rasht City. Thirtyfour people were selected and studied as samples based on entry and exit criteria, admission and exit criteria, and before the outbreak of the Covid-19 in January 2019. However, during one year of the study, due to coronary heart disease and death, the samples were reduced to 26, and none of them were infected with the coronavirus. Inclusion criteria included were as follow: age range 60 to 74 years and obtaining a score of 24 or higher in the mental status test.

Exclusion criteria included assistive devices, coronary heart disease, hearing and vision problems, amputation due to illness and problems. An effective temporary on the test day was a history of severe physical and muscular illness and dependence on wheelchairs, which was collected using medical records and questions from nursing home staff.

The subjects were evaluated by the health protocols related to coronary heart disease and with the approval of Guilan Province Welfare Office and the officials of the relevant centers. Also, a code of ethics was received from the Ethics Committee of Guilan University of Medical Scienc- es. Evaluation of the motor performance of the elderly with berg functional balance tests, Time Up and Go test or fall probability and walking speed test or 10-meter walk, was performed in two stages once in January 2019 and then one year later in 2020. Shapero-Wilk test was used to check the normality of the data, descriptive statistics were used to determine the mean and standard deviation, and paired t-test and McNemar tests were used in SPSS software version 26 to analyze the data and examine the intra-group differences between the data.

\section{Results}

The results of the correlated t-test showed that the time up and go test in the elderly under the influence of one year of movement limitation in the post-coronary period had a significant increase $(\mathrm{P}=0.001)$, also in functional balance index $(\mathrm{P}=0.03)$ significant changes according to For a year, the elderly had limited mobility. There was a significant decrease in the walking variable $(\mathrm{P}=0.001)$ following this period. It also showed a significant increase in the probability of falling index $(\mathrm{P}=0.021)$.

\section{Discussion and Conclusion}

According to the findings, the motor function of the elderly has decreased during this one year, and the care centers and the families of the elderly should pay close attention to the exercise and physical activity in this critical period of the pandemic. Encourage the elderly to participate in sports activities to reduce inactivity. Also, the officials of the elderly care centers should take the necessary measures to implement sports programs for this segment of the society in consultation with specialized and competent people.

The limitations of this study include the limited number of samples, the lack of a control group due to the prevalence of coronary pandemic and its complications in the level of physical activity of all the elderly, physical changes due to aging and uncontrolled mental and psychological conditions of subjects. It is suggested that in future studies, the effects of coronary restrictions on the physical activities of other specific individuals (Parkinson, MS, etc.) and with a larger sample size be investigated. This research has not received any financial support from public, private and nonprofit organizations. 


\section{Ethical Considerations}

Compliance with ethical guidelines

All ethical principles are considered in this article. The participants were informed about the purpose of the research and its implementation stages. They were also assured about the confidentiality of their information. They were free to leave the study whenever they wished, and if desired, the research results would be available to them. This study was approved by the Research Ethics Committee of the Guilan University of Medical Sciences (Code: IR.GUMS.REC.1399.252).

Funding

This article has been done with research support between the University of Guilan and the General Office of Sports and Youth of Guilan Province.

Authors' contributions

The authors contributed equally in preparing this article.

Conflict of interest

The authors declared no conflict of interest.

Acknowledgments

The authors would like to thank the General Department of Welfare of the Province Guilan and the City of Rasht. 
تأثير يكى سال كمتحركى ناشى از كوويد 19 بر عملكرد حركتى سالمندان ساكن در مراكز مراقبتى

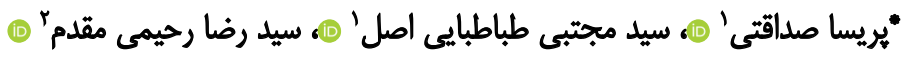

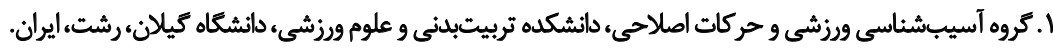

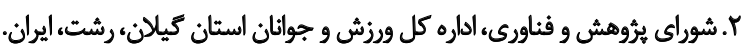

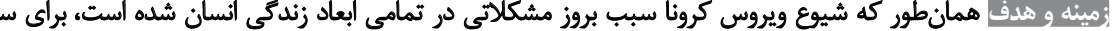

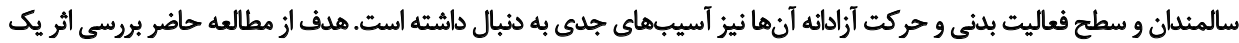

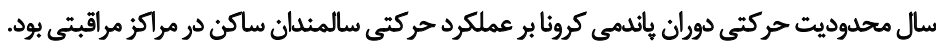

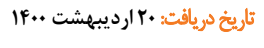

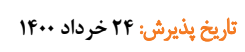

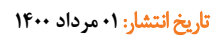

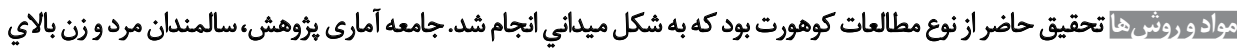

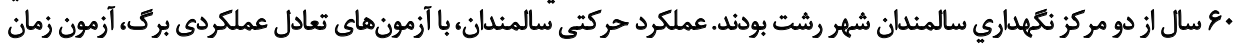

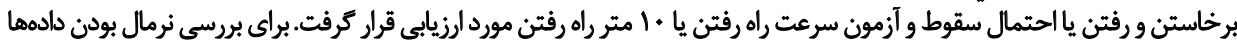

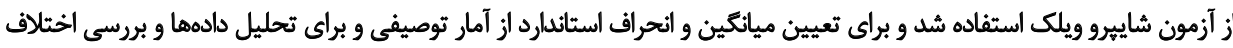

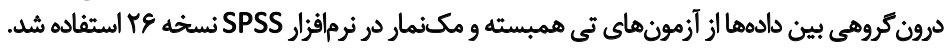

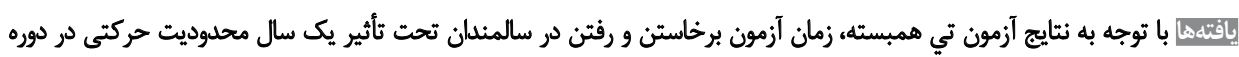

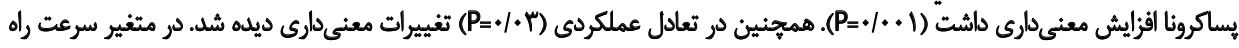

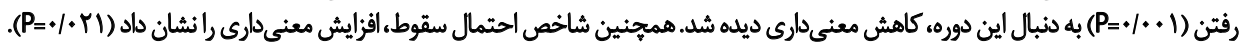

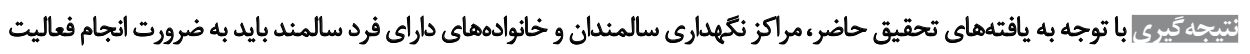

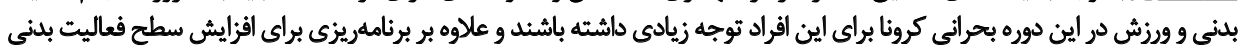

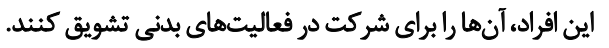

كليدواؤه هاء:

ويروس كرونا، تعادل،

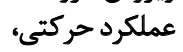
احتمال سقوط إكني

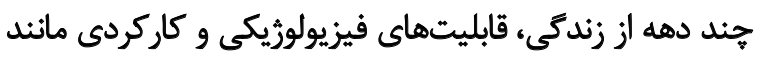

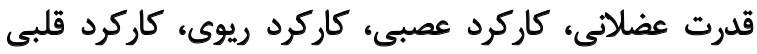

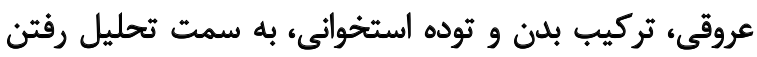

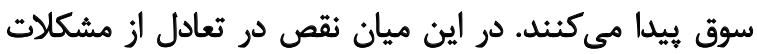

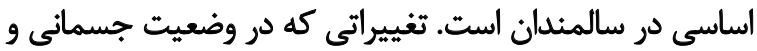

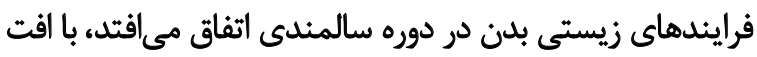

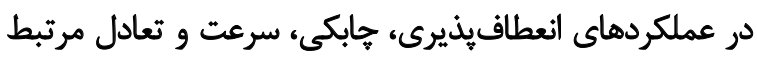

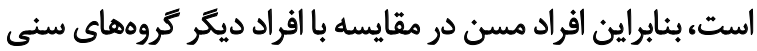

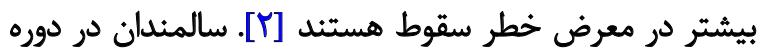

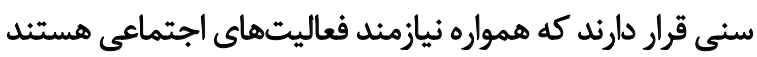

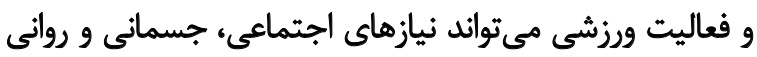

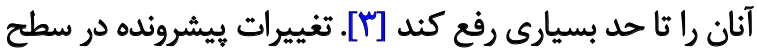

daco سالمندى دوران حساسى از زندكى است و با در نظر داشتن

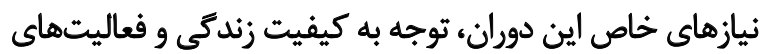

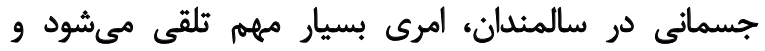

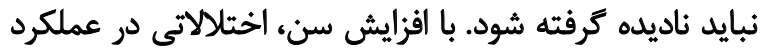

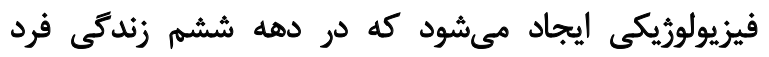

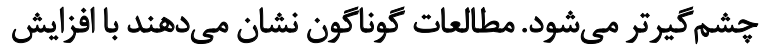

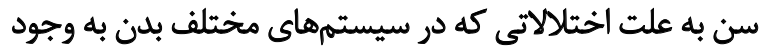

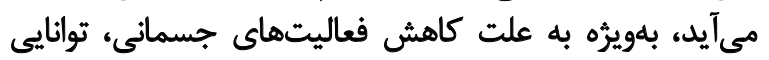

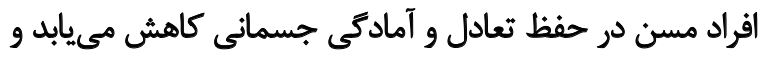

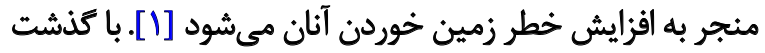


اسكارتونى و همكاران در تحقيقى به بررسى اثر ورزش بر

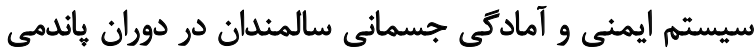

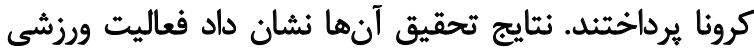

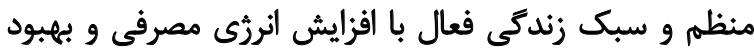

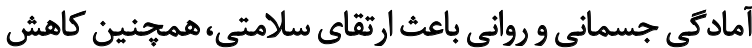

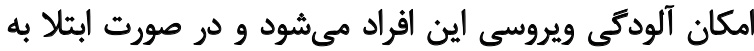

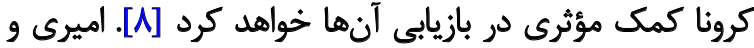

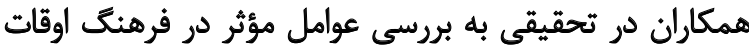

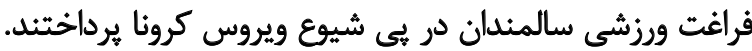

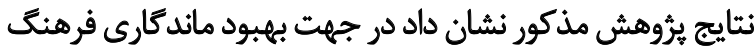

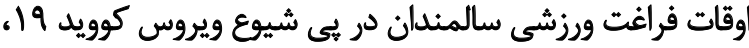

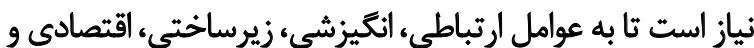
بهداشتى توجه ويرٔه شود [9].

ازآنجاييى كه ابعاد مختلف بيامدهاي بيمارى كرونا در جامعه

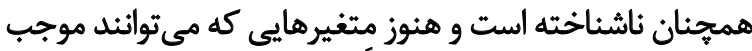

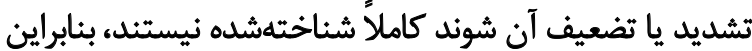

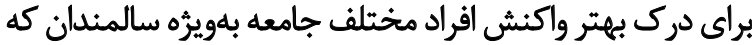

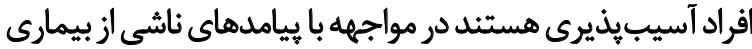

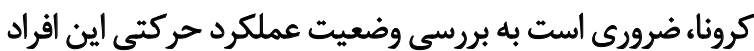

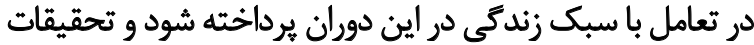

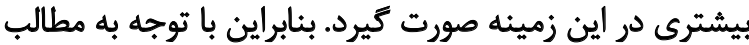

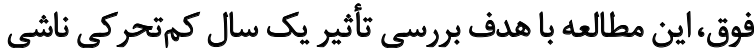

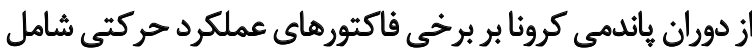

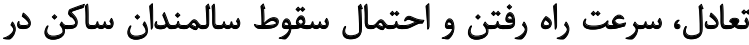

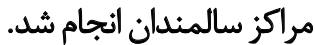

\section{مواد و روشئها}

تحقيق حاضر از نوع مطالعات كوهورت بود كه به شكل ميداني

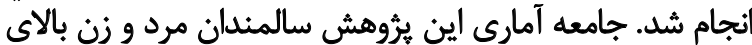

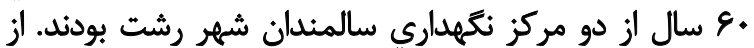

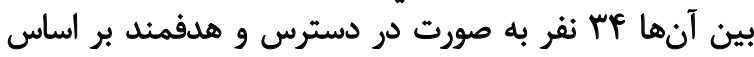

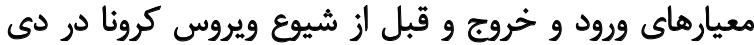

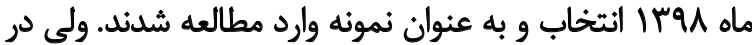

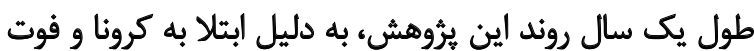

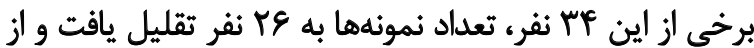

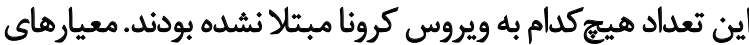

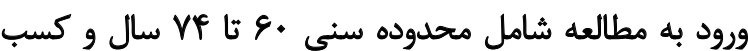

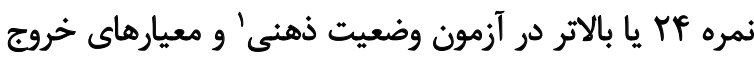

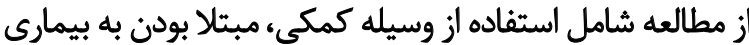

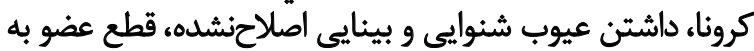

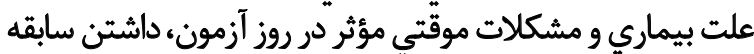
بيماري شديد جسماني و عضلاني و وابستكي افراد بـاد به صندلي

\section{Mini-Mental State Examination (MMSE)}

جامعه سبب شده است تا اشكال مختلفى از فعاليتهاى جسمانى و اوقات فراغت به صورت غيرفعال شكل كيرد كه ائه اين مسئله

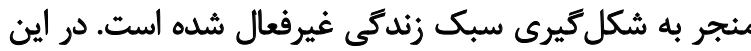

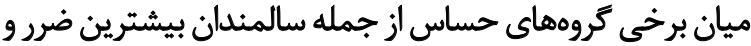

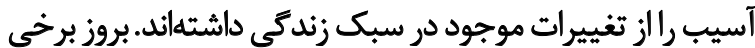

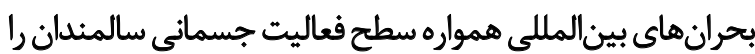

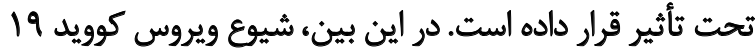

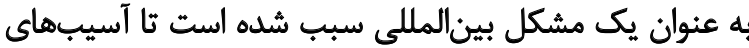
جدى بر آمادگى جسمانى و ورزش سالمندان در سطح جهان

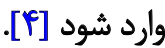

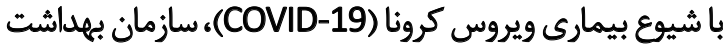

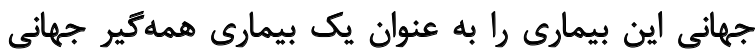

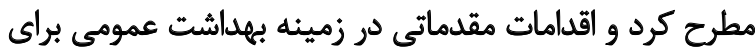

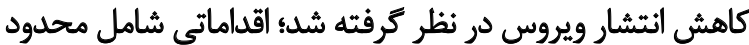

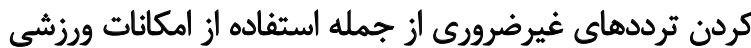

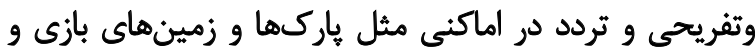

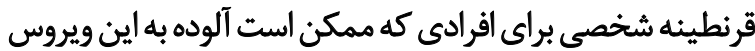

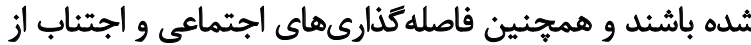

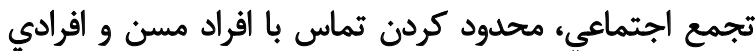

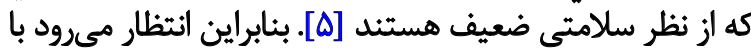

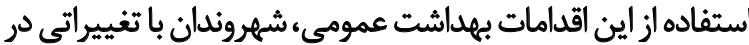

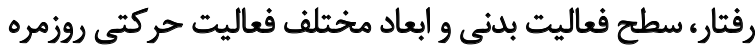

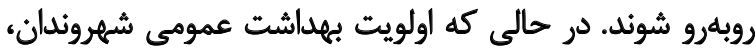

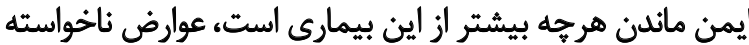

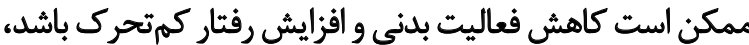

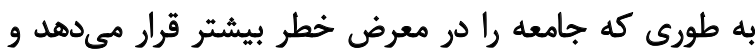

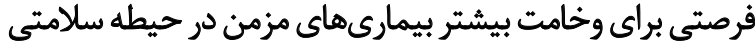

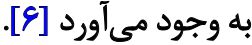

همان طور كه شيوع ويروس كوويد 19 سبب بروز مشكلاتى در

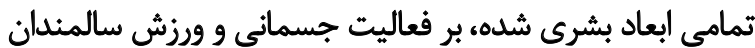

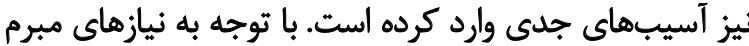

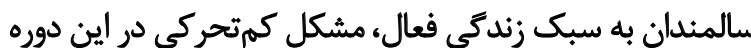

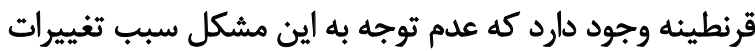

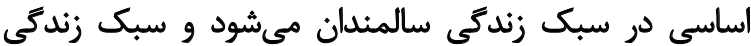

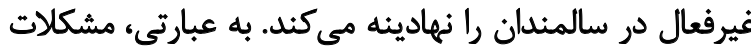

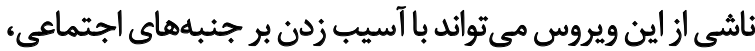

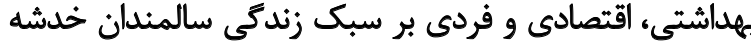

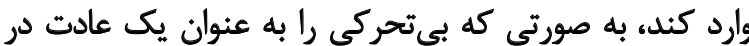

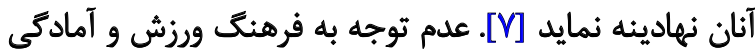

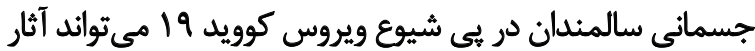

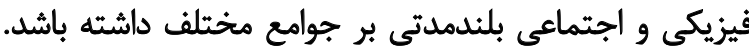

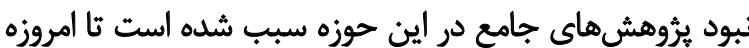

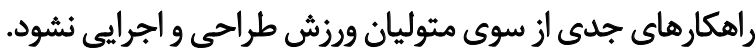


ارزيابي احتمال سقوط: براى ارزيابي احتمال سقوط از آزمون

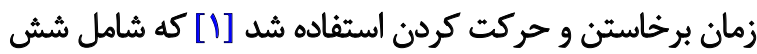

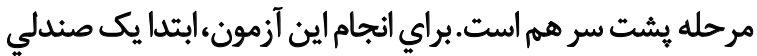

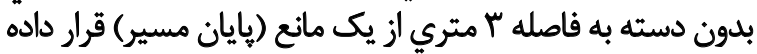

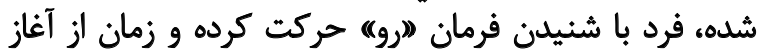

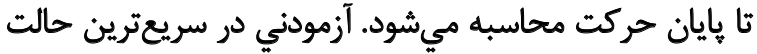

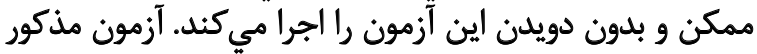

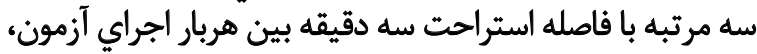

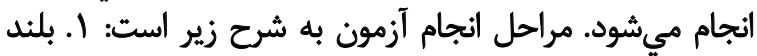

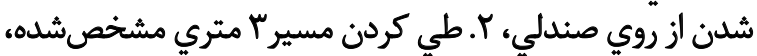

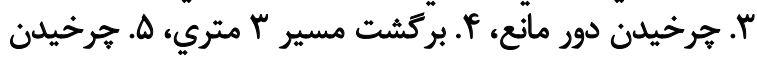

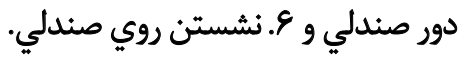

نحوه امثيازكذاري: مدتزماني كه آزمودني بتواند اين آزمون

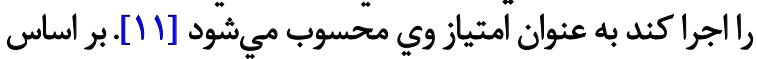

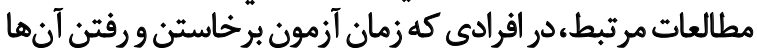

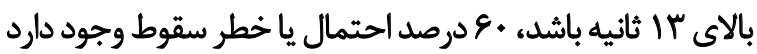

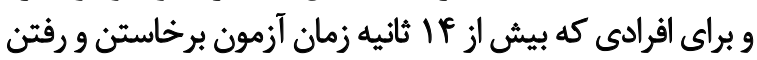

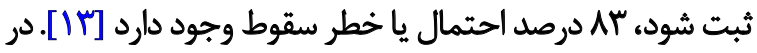

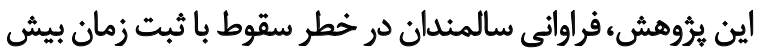

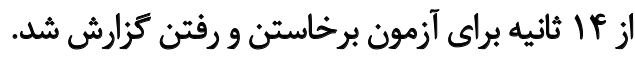

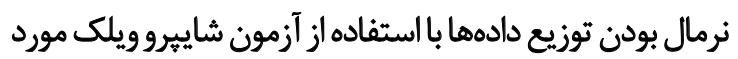

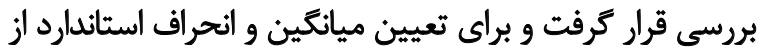

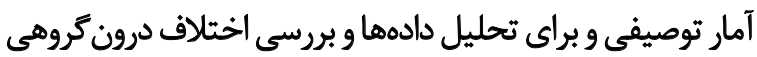

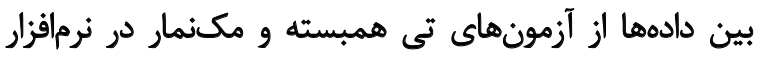
SPSS

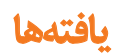

مشخصات جمعيتشناختى سالمندان شركت كنينده در اين مطالعه به شرح جدول شماره 1 است.

در جدول شماره r ميانگين و انحراف استاندارد عملكرد

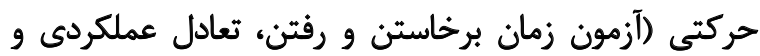

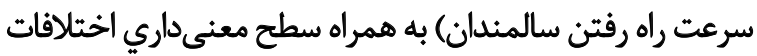

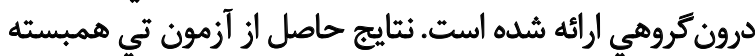

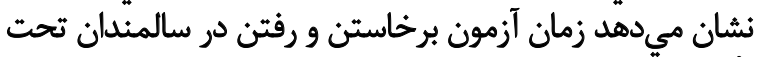

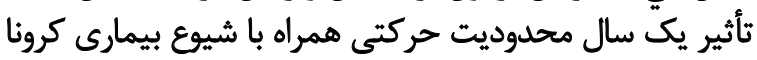

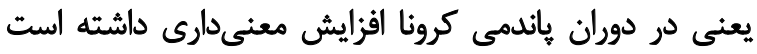

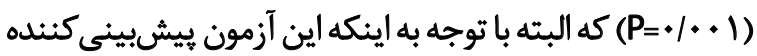

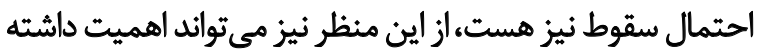

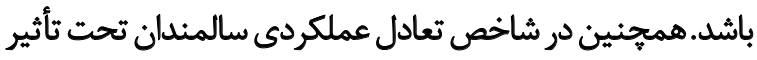

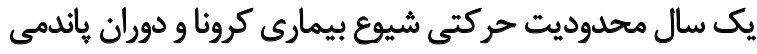

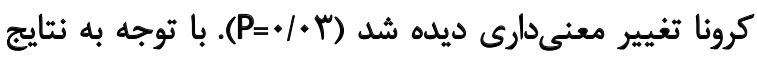

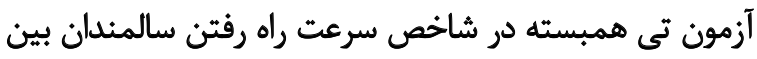

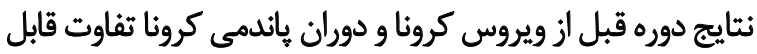

جرخدار بود كه با استفاده از اطلاعات برونده يزشكى و سؤال

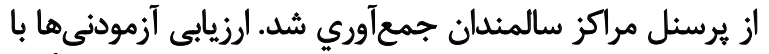

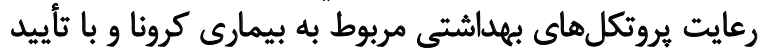

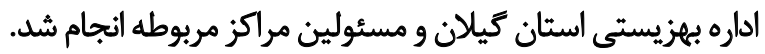

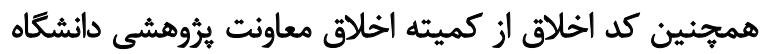

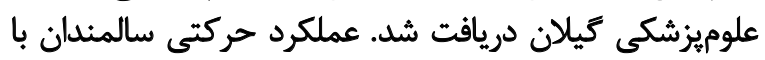

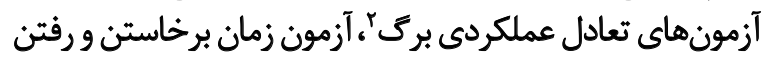

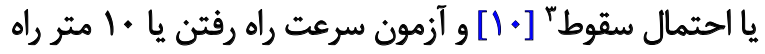

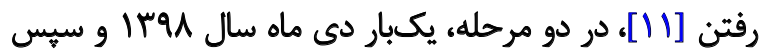

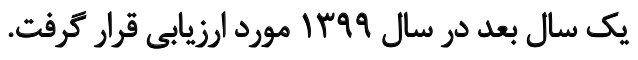

آزمون ارزيابى وضعيت ذذهنى (MMSE): اين آزمون با توجيه

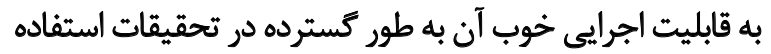

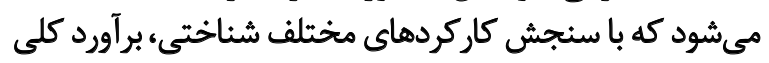

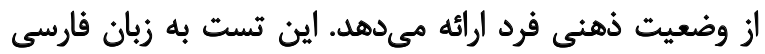

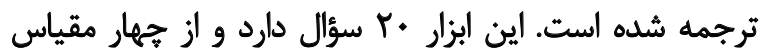

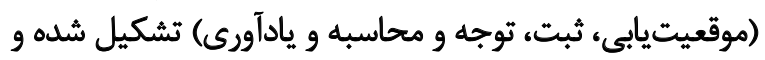

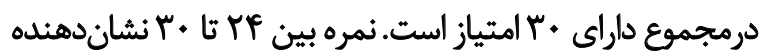

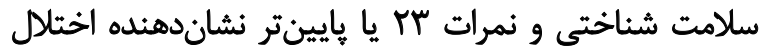
شناختى در نظر كرفته مي وشود [IT]

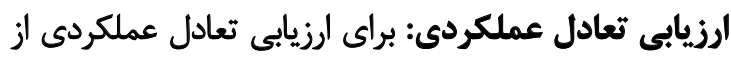

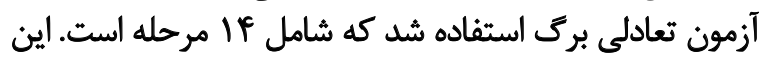

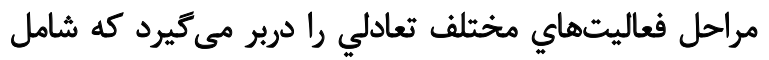

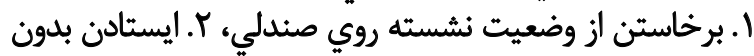

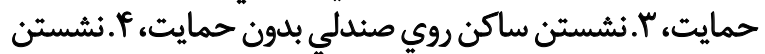

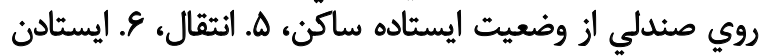

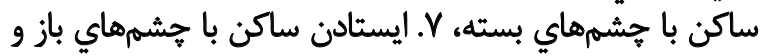

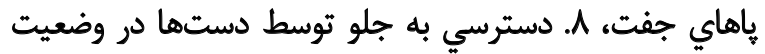

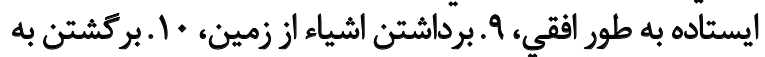

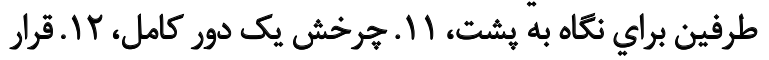

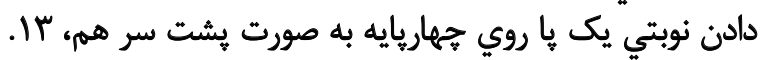

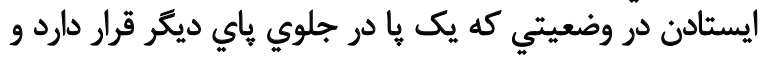

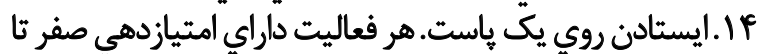

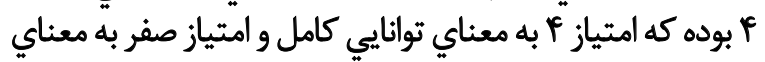

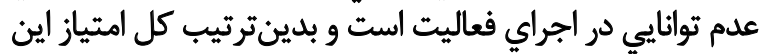

آزمون وها أست [-1].

آزمون •ا متر راه رفتن (سرعت راه رفتن): از آزمودنيها

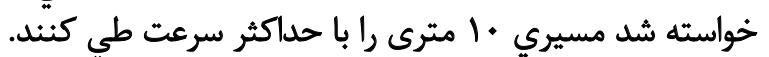

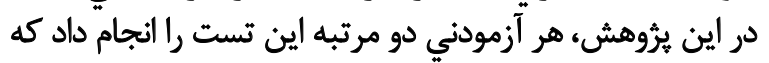

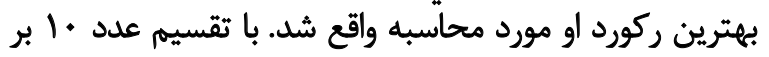

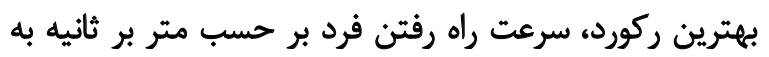

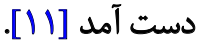

3. Timed Up and Go test (TUG test) 
جدول (. مشخصات جمعيتشناختى آزمودنىها (n=r\&)

\begin{tabular}{|c|c|c|c|}
\hline هداكثر & 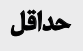 & ميانكين داتحراف معيار & مشخصات \\
\hline$n$ & er & EN/ITN & سن تقويمى (سال) \\
\hline$n$ & $\Delta F$ & $8 \Delta / \tau \pm r / q$ & وزن (كيلوكرم) \\
\hline ine & laV & $\mid \varnothing \varnothing / r \pm ץ / 1$ & قد (سانتىمتر) \\
\hline$\pi$ & $\pi$ & $m / 11 \pm 1 /$. & شاخص توده بدنى (كيلوكرم بر متر مربع) \\
\hline
\end{tabular}

كرد كه به بررسى اثر محدوديتهاى بيمارى كرونا بر فعاليت

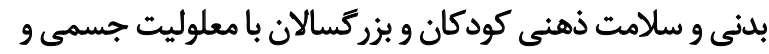

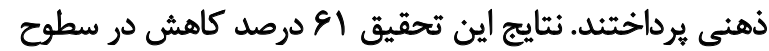

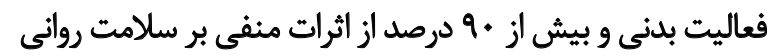

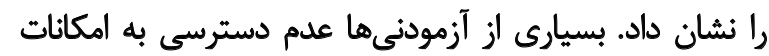

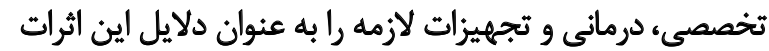

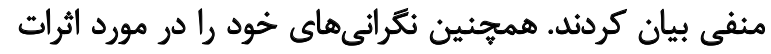

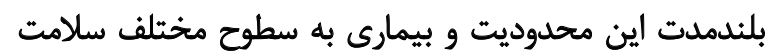

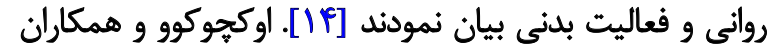

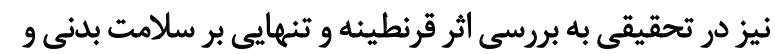

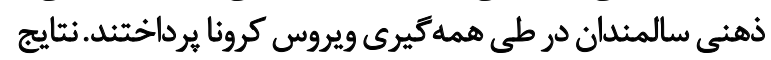

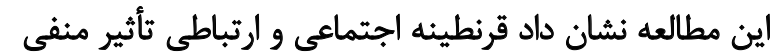

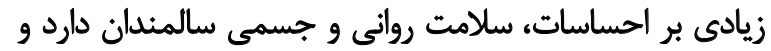

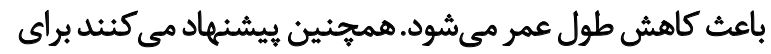

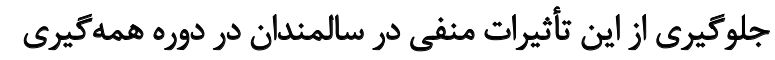

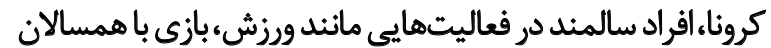

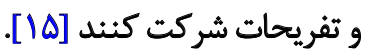

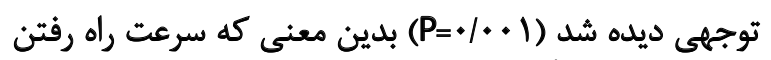

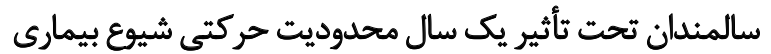
كرونا دجار كاهش شده است.

\section{ثִ}

مطالعه حاضر با هدف بررسى اثر يك سال محدوديت حركتى

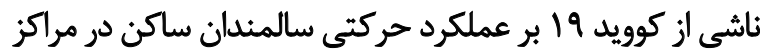

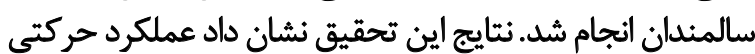

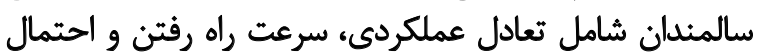

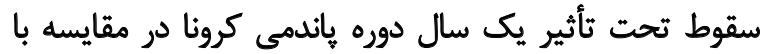

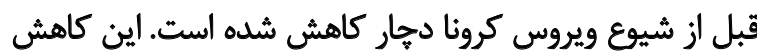

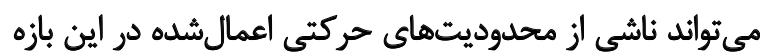

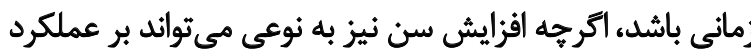

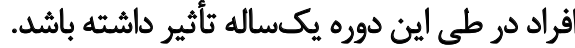

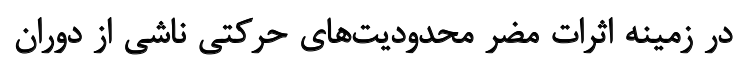

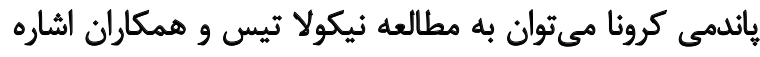

جدول r. نتايج مقايسه عملكرد حركتي سالمندان ساكن در مراكز سالمندان در دوران هاندمي كرونا

\begin{tabular}{|c|c|c|c|c|c|c|}
\hline $\mathbf{P}$ & $\mathbf{t}$ & df & اختلاف ميانكين & ميانكين +|ثحراف معيار & مراحل آزمون & متغير \\
\hline $.1 . .1^{*}$ & $\varphi \cdot 1$ & ro & $1 / 4$. & $\begin{array}{l}1 F / \& V \pm 1 / .9 \\
10 / M \pm 1 / K A\end{array}$ & دوبل از كرونا & أزمون برخاستن و رفتن \\
\hline 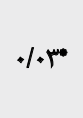 & $r / M$ & ro & $+N 8$ & $\begin{array}{l}r V / r+ \pm 1 / q F \\
r g / \Delta r \pm 1 / q .\end{array}$ & قوربل از كرونا & تعادل عملكردى \\
\hline $.1 .01^{*}$ & e/pe & ra & $1 / 81$ & 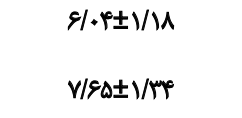 & دورة قبل كرونا & زمان ما هثر راله رقفن (ثانيه) \\
\hline $.1 .01^{*}$ & $r / 99$ & ro &.$/ 48$ & $\begin{array}{l}W M \pm \cdot M \\
W / M \pm \cdot M\end{array}$ & دبل از كرونا & سرعت راه رفتن (مثر بر \\
\hline & & $A+r \mid *$ & & 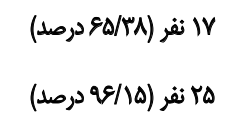 & دوريل از كرونا & احتمال سقوط (فراوانى) \\
\hline
\end{tabular}


در برخى از سيستمهاء مى توان اين مشاورهها را با استفاده از

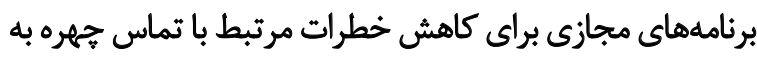

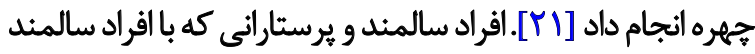

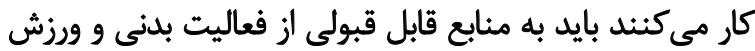

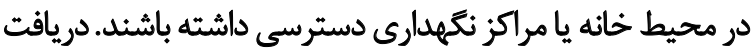

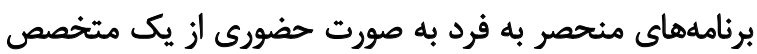

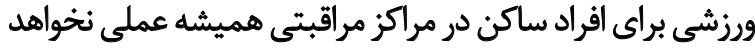

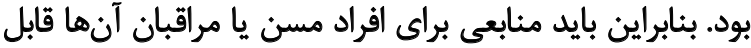

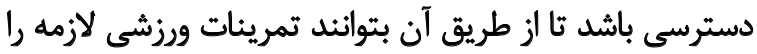

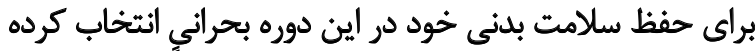

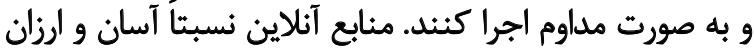

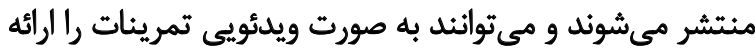

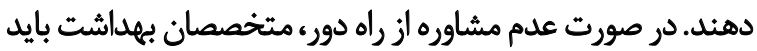
از لحاظ كردن اين خدمات حمايت كنيند تاافراد سالمند همجندان

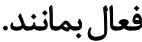

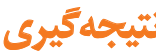

با توجه به يافتهاي تحقيق حاضر، عملكرد حركتى سالمندان

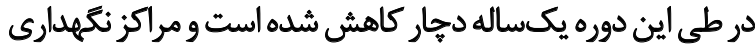

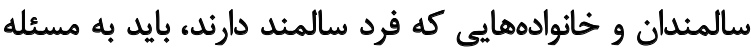

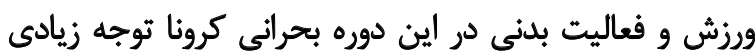

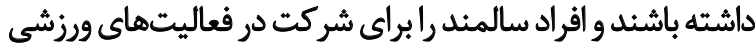

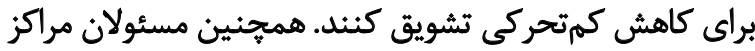

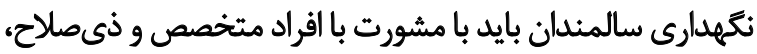

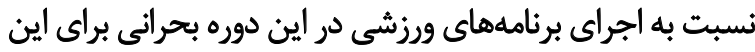
قشر از جامعه اقدامات لازم را به عمل آورند.

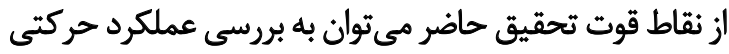

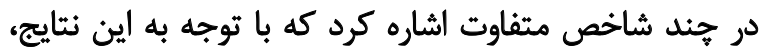

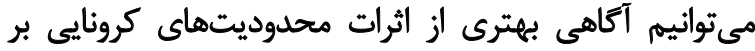

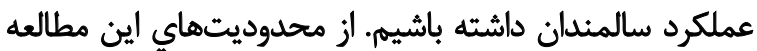

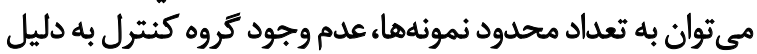

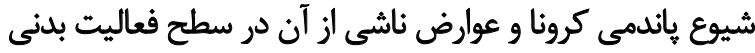

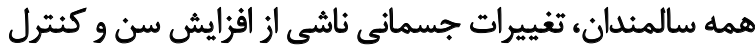

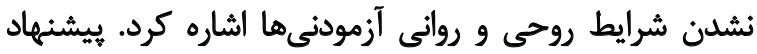

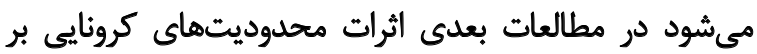

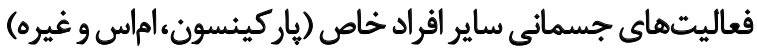
و با حجم نمونه بيشتر مورد بررسى قرار كيرد.
افراد سالمند با مشكلات باليني قابل توجه يا محدوديتهاي

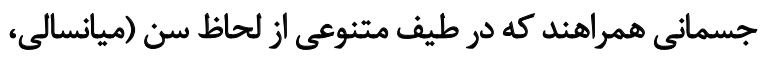

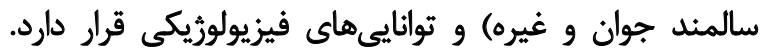

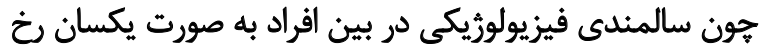

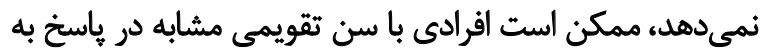

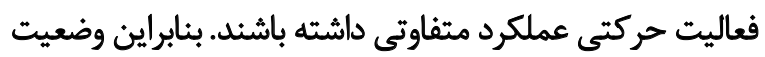

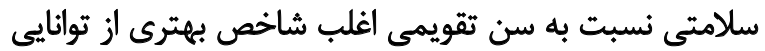

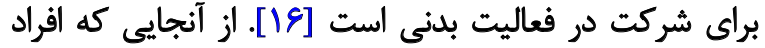

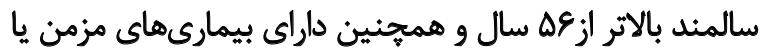

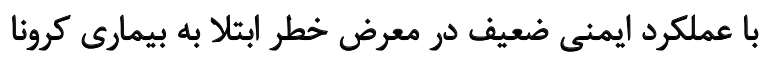

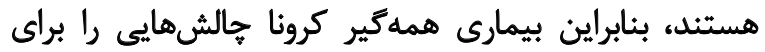

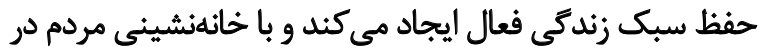

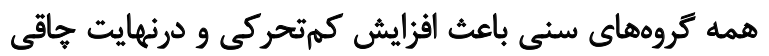

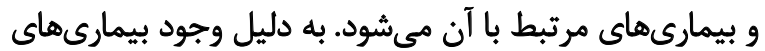

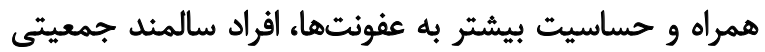

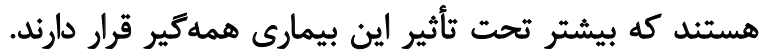

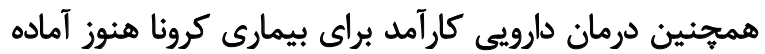

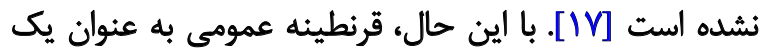

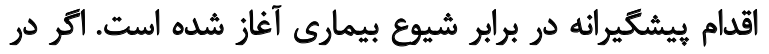

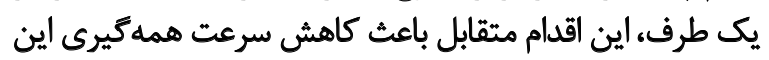

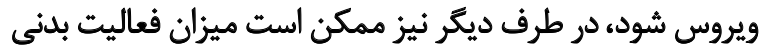

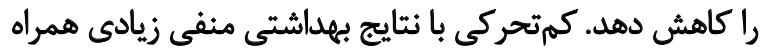

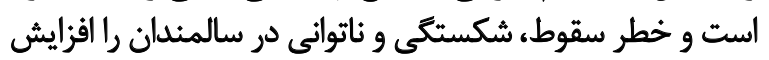

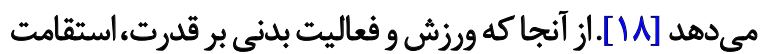

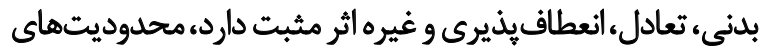

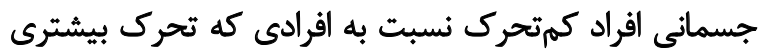

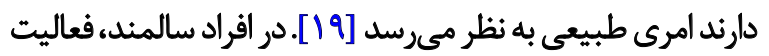

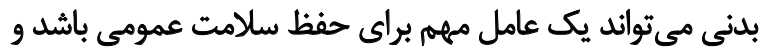

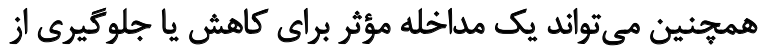

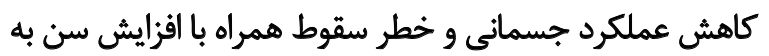

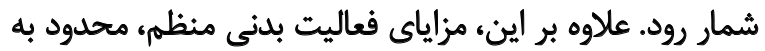

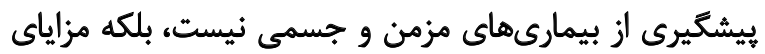

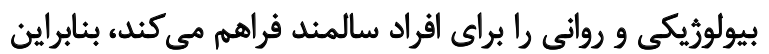

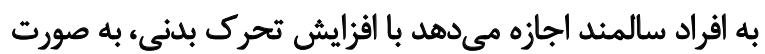

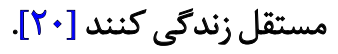
توصيdهاى يك متخصص سلامت و بهداشت مى تواند تعداد

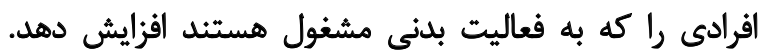

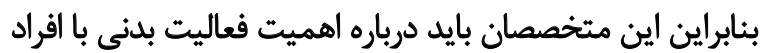

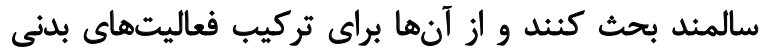

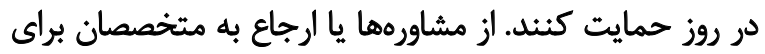

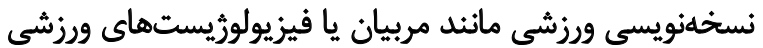

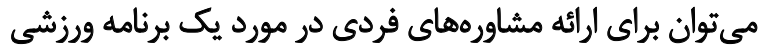

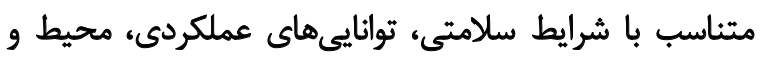
ترجيحات و اهداف شخصى يك فرد سالمئد استفاده كرد. 


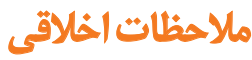

بيروى ازٔ اصول الخاق يثروهش

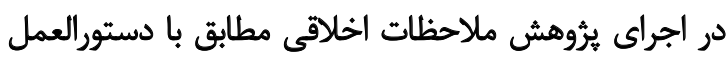

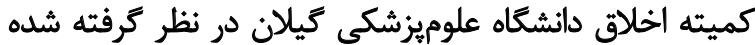

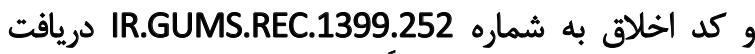

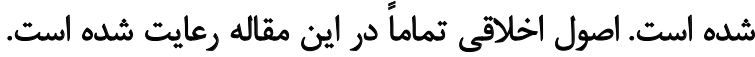

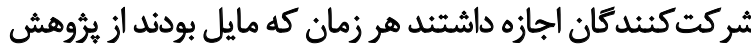

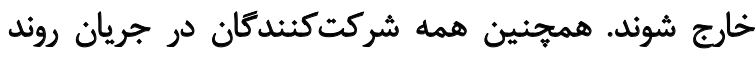

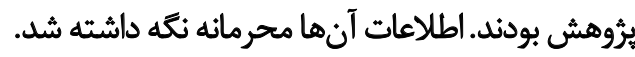

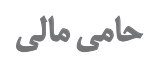

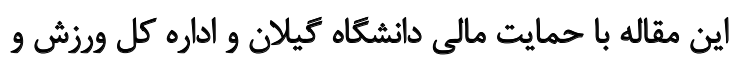
جوانان استان كيلان انجام شده است.

$$
\text { مشاركت نويسئدكًان }
$$

تمام نويسندكان در آمادهسازى اين مقاله مشاركت يكسان

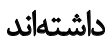

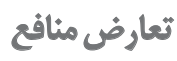

بنابر اظهار نويسندكان، اين مقاله تعارض منافع ندارد.

$$
\text { تشكر و قدرواني }
$$

بدينوسيله از مسئولين محترم اداره كل بهزيستى استان كيلان و شهر رشت تشكر و قدردانى مى مودمد ادره 


\section{References}

[1] Koohboomi M, Norasteh AA, Samami N. [Effect of Yoga training on physical fitness and balance in elderly females (Persian)]. Iranian Journal of Ageing. 2015; 10(3):26-35. https://www.sid. ir/en/journal/ViewPaper.aspx?ID=580064

[2] Lin HW, Bhattacharyya N. Balance disorders in the elderly: Epidemiology and functional impact. The Laryngoscope. 2012; 122(8):1858-61. [DOI:10.1002/lary.23376] [PMID]

[3] Park S, Lee HJ, Jeon BJ, Yoo EY, Kim JB, Park JH. Effects of occupational balance on subjective health, quality of life, and health-related variables in community-dwelling older adults: A structural equation modeling approach. Plos One. 2021; 16(2):e0246887. [DOI:10.1371/journal.pone.0246887] [PMID] [PMCID]

[4] Hammami A, Harrabi B, Mohr M, Krustrup P. Physical activity and coronavirus disease 2019 (COVID-19): Specific recommendations for home-based physical training. Managing Sport and Leisure. 2020. [DOI:10.1080/23750472.2020.1757494]

[5] Freeman S, Eykelbosh A. COVID-19 and outdoor safety: Considerations for use of outdoor recreational spaces. National Collaborating Centre for Environmental Health. 2020. https:// ncceh.ca/documents/guide/covid-19-and-outdoor-safety-considerations-use-outdoor-recreational-spaces

[6] Chen P, Mao L, Nassis GP, Harmer P, Ainsworth B, Li F. Returning Chinese school-aged children and adolescents to physical activity in the wake of COVID-19: Actions and precautions. Journal of Sport and Health Science. 2020; 9(4):322-4. [DOI:10.1016/j. jshs.2020.04.003] [PMID] [PMCID]

[7] Son JS, Nimrod G, West ST, Janke MC, Liechty T, Naar JJ. Promoting older adults' physical activity and social well-being during COVID-19. Leisure Sciences. 2021; 43(1-2):287-94. [DOI:10.108 0/01490400.2020.1774015]

[8] Scartoni FR, Sant'Ana LD, Murillo-Rodriguez E, Yamamoto T, Imperatori $\mathrm{C}$, Budde $\mathrm{H}$, et al. Physical Exercise and Immune System in the Elderly: Implications and importance in COVID-19 pandemic period. Frontiers in Psychology. 2020; 11:593903. [DOI:10.3389/fpsyg.2020.593903] [PMID] [PMCID]

[9] Amiri SM, Jamshidain L, Torkfar A, Arvin H. [Factors affecting the persistence of sports culture in the elderly following the outbreak of Covid-19 virus (Persian)]. Research on Educational Sport. 2021; 8(21):139-60. [DOI:10.22089/res.2020.9225.1928]

[10] TabatabaiasI SM, Sedaghati P, Javazi F. [Comparison of functional balance and probability of falling in the elderly with and without a history of falling living in care centers (Persian)]. Journal of Sport Biomechanics. 2020; 6(2):134-43. [DOI:10.32598/ biomechanics.6.2.6]

[11] Lopopolo RB, Greco M, Sullivan D, Craik RL, Mangione KK. Effect of therapeutic exercise on gait speed in community-dwelling elderly people: A meta-analysis. Physical Therapy. 2006; 86(4):520-40. [DOI:10.1093/ptj/86.4.520] [PMID]

[12] Trzepacz PT, Hochstetler H, Wang S, Walker B, Saykin AJ. Relationship between the montreal cognitive assessment and mini-mental state examination for assessment of mild cognitive impairment in older adults. BMC Geriatrics. 2015; 15:107. [DOI:10.1186/s12877-015-0103-3] [PMID] [PMCID]
[13] Zakaria NA, Kuwae Y, Tamura T, Minato K, Kanaya S. Quantitative analysis of fall risk using TUG test. Computer Methods in Biomechanics and Biomedical Engineering. 2015; 18(4):426-37. [DOI:10.1080/10255842.2013.805211] [PMID]

[14] Theis N, Campbell N, De Leeuw J, Owen M, Schenke KC. The effects of COVID-19 restrictions on physical activity and mental health of children and young adults with physical and/or intellectual disabilities. Disability and Health Journal. 2021; 14(3):101064. [DOI:10.1016/j.dhjo.2021.101064] [PMID] [PMCID]

[15] Okechukwu CE. The impact of loneliness on physical and mental health among older adults in the era of coronavirus disease 2019 pandemic. Apollo Medicine. 2021; 18(1):29-32. [DOI:10.4103/am.am_3_21]

[16] Garber CE, Blissmer B, Deschenes MR, Franklin BA, Lamonte MJ, Lee IM, et al. Quantity and quality of exercise for developing and maintaining cardiorespiratory, musculoskeletal, and neuromotor fitness in apparently healthy adults: Guidance for prescribing exercise. Medicine \& Science in Sports \& Exercise. 2011; 43(7):1334-59 [DOI:10.1249/MSS.0b013e318213fefb]

[17] Narici M, De Vito G, Franchi M, Paoli A, Moro T, Marcolin G, et al. Impact of sedentarism due to the COVID-19 home confinement on neuromuscular, cardiovascular and metabolic health: Physiological and pathophysiological implications and recommendations for physical and nutritional countermeasures. European Journal of Sport Science. 2020; 21(4):614-35. [DOI:10. 1080/17461391.2020.1761076] [PMID]

[18] Moro T, Paoli A. When COVID-19 affects muscle: Effects of quarantine in older adults. European Journal of Translational Myology. 2020; 30(2):9069. [DOI:10.4081/ejtm.2020.9069]

[19] Sadrollahi A, Hosseinian M, Alavi NM, Khalili Z, Esalatmanesh S. Physical activity patterns in the elderly kashan population. Iranian Red Crescent Medical Journal. 2016; 18(6):e25008. [DOI:10.5812/ircmj.25008]

[20] Ghram A, Briki W, Mansoor H, Al-Mohannadi AS, Lavie CJ, Chamari K. Home-based exercise can be beneficial for counteracting sedentary behavior and physical inactivity during the COVID-19 pandemic in older adults. Postgraduate Medicine. 2020; 133(5):469-80. [DOI:10.1080/00325481.2020.1860394] [PMID]

[21] Said CM, Batchelor F, Duque G. Physical activity and exercise for older people during and after the coronavirus disease 2019 pandemic: A path to recovery. Journal of the American Medical Directors Association. 2020; 21(7):977-9. [DOI:10.1016/j. jamda.2020.06.001] [PMID] [PMCID] 